\title{
BRAIN TUMORS
}

\section{RADIOTHERAPY FOR LOW-GRADE ASTROCYTOMAS}

The effect of radiotherapy on the survival of 143 children with lowgrade (grade 1 or 2) astrocytomas was evaluated at the Royal Manchester Children's Hospital, and Christie Hospital and Holt Radium Institute, Manchester, UK. The 5-year survival was $85 \%, 10$-year $79 \%$. and 15 -year $72 \%$. Children over 3 years of age had a better prognosis than younger children. Peripherally located tumors, with their easier access and more complete resection, have a significantly better survival outcome than deep-seated tumors. In 68 children whose tumors were completely resected and who required no radiation, the 15-year survival rate was 90\%; if radiotherapy was given for some reason the 15 -year survival fell to $75 \%$ for this peripheral tumor group. All 9 children with deep-seated tumors died when treated with surgery alone; a 15 -year survival rate of $64 \%$ was obtained in patients who received radiation after surgery for deep tumors. (West CGH et al. Radiotherapy in the treatment of low-grade astrocytomas. I. A survival analysis. Child's Nerv Syst August 1995;11:438-442). (Respond: Mr Charles GH West, Department of Neurological Surgery, Royal Manchester Children's Hospital, Manchester M27 1HA, UK).

COMMENT. Radiation therapy had no benefit and in some cases, an adverse effect in children with superficial tumors that were amenable to complete or near complete resection. In deep seated tumors incompletely resected, radiation after surgery resulted in a significant improvement in survival rates. While radiotherapy may improve the survival rate in children with deep tumors, it can adversely affect the quality of life by causing long-term cognitive dysfunction.

\section{RADIOTHERAPY-INDUCED COGNITIVE SEQUELAE}

Long-term neurological and neuropsychological outcome in 25 irradiated children $<15$-years of age and 25 treated with surgery alone for lowgrade astrocytoma was evaluated by two neurosurgeons and two psychologists independently at the Royal Manchester Children's Hospital, UK. Neurological function was not different in the irradiated group compared to the nonirradiated patients. In all neuropsychological tests used to assess intelligence, word reading, memory, learning and information processing, the performance of children with cranial radiation therapy was worse than those treated by surgery alone, and significant changes occurred in tests of IQ and information processing. Special education was required more frequently in the irradiated group. Both supratentorial tumor radiotherapy and local field irradiation to the posterior fossa for cerebellar tumors produced significant cognitive impairments. (Chadderton RD et al. Radiotherapy in the treatment of low-grade astrocytomas. II. The physical and cognitive sequelae. Child's Nerv Syst August 1995;11:443-448). (Respond: Mr CGH West, Department of Neurological Surgery, Royal Manchester Children's Hospital, Manchester M27 1HA, UK).

COMMENT. This study confirms previous reports of cognitive impairments following irradiation for supratentorial tumors in children. Additionally, even local field irradiation to the posterior fossa can result in learning and academic problems. Children receiving cranial radiation therapy, locally or to the whole brain, should be followed with neuropsychological testing for longer than 3 years to 
determine effects on IQ and the need for special education.

\section{INTRACRANIAL EPENDYMOMA FOLLOW-UP STUDY}

The management and outcome of 24 children with intracranial ependymomas ( 22 benign and well differentiated) treated over a 10-year period, 1979-1988, and with a minimum 5-year follow-up were analysed at Great Ormond Street Hospital, London, UK. Of 16 with infratentorial ependymomas, 4 were alive, all 4 having total resection and 3 having craniospinal radiotherapy in addition. Of 12 who died, 5 had total resections and 4 had radiotherapy. All 7 with incomplete resections had died within 5 years despite radiotherapy and chemotherapy as well in some. Ventriculoperitoneal shunting for hydrocephalus was required in 8 of 17 patients with posterior fossa ependymomas. In the supratentorial group of 7 patients, only one was alive and tumor-free at 5 years. No metastases were detected by myelography in 9 patients examined. (Jayawickreme DP, Hayward RD, Harkness WFJ. Intracranial ependymomas in childhood: a report of 24 cases followed for 5 years. Child's Nerv Syst July 1995;11:409-413). (Respond: Mr RD

Hayward, Department of Neurosurgery, Great Ormond Street Hospital, Great Ormond Street, London WC1 3JH, UK).

COMMENT. In posterior fossa ependymomas an apparent total resection is associated with a better outcome but may be followed by local recurrence. Partial resection carries a very poor outcome, even with radiotherapy. Supratentorial ependymomas have a worse prognosis than the infratentorial group. In general, intracranial ependymomas in childhood have a very poor prognosis. Long-term survival may be achieved only after complete resection followed by radiotherapy, with its known effects on intellectual function and school achievement. Spinal metastases are unusual and prophylactic spinal irradiation in benign ependymoma is not recommended. Multicenter studies including chemotherapy are indicated.

\section{DEVELOPMENTAL DISABILITIES}

\section{NEUROLOGICAL ASSESSMENT OF DEVELOPMENTAL DELAY}

The etiologic or diagnostic yield of the neurologic examination in 60 children referred to a pediatric neurologist for evaluation of global developmental delay was determined at the Montreal Children's HospitalMcGill University, Quebec, Canada. Examination at a mean age of 3.58 years revealed mild delay in development in 25 , moderate delay in in 23 , and severe delay in 12. EEG, MRI, metabolic screens, fragile $X$ test and karyotype established an etiologic diagnosis in 38 (63\%), including cerebral dysgenesis (17\%), HIE (10\%), chromosomal anomalies (10\%), toxins $(8 \%)$, and metabolic disorders (5\%). (Majnemer A, Shevell MI. Diagnostic yield of the neurologic assessment of the developmentally delayed child. I Pediatr August 1995;127:193199). (Reprints: Annette Majnemer PhD, Montreal Children's Hospital, 2300 Tupper St, Room A-509, Montreal, Quebec H3H 1P3, Canada).

COMMENT. Optimal management of children with developmental delay should include a neurologic examination and selected laboratory tests. An etiologic diagnosis provides physician and family with important information regarding risks of recurrence and choice of therapeutic intervention. 